Revista de Biología Marina y Oceanografía

Vol. 48, №3: 641-645, diciembre 2013

DOI 10.4067/S0718-19572013000300021

Nota Científica

\title{
Efecto combinado de la temperatura y salinidad sobre la supervivencia embrionaria de Galaxias maculatus
}

\author{
Combined effect of temperature and salinity on embryonic survival of Galaxias maculatus
}

\author{
Juan Barile ${ }^{1}$, Manuel Escudero ${ }^{1}$ y Eriko Carreño ${ }^{1}$
}

\begin{abstract}
'Escuela de Acuicultura, Universidad Católica de Temuco, Av. Rudecindo Ortega 02950 Temuco, Chile. jbariles@uct.cl
Abstract.- This study aims to evaluate the effect of temperature and salinity on Galaxias maculatus embryo survival. Embryos were incubated at 5,10 and $15^{\circ} \mathrm{C}$ and 0,10 and 30 psu. At $5^{\circ} \mathrm{C}$ all salinities induced $100 \%$ mortality. Furthermore, salinity of 30 , regardless temperature, caused $100 \%$ mortality. In freshwater, embryos reached 74.7 and $69.3 \%$ survival at $10^{\circ} \mathrm{C}$ and $15^{\circ} \mathrm{C}$, respectively. While, at $10 \mathrm{psu}$, the larvae survivals were higher, 88.7 and $80.0 \%$ with 10 and $15^{\circ} \mathrm{C}$ respectively. These findings suggest that there is not a temperature-salinity interaction effect on embryonic development of $\mathrm{G}$. maculatus. In conclusion the embryonic development of this fish is only possible at fresh and brackish waters and it is not viable at salt water.
\end{abstract}

Key words: Environmental parameters, fish, embryos, Galaxias maculatus

\section{INTRODUCCIÓN}

El desarrollo embrionario del puye Galaxias maculatus (Jenyns, 1842), fue descrito detalladamente hace más de 40 años por Benzie (1968). A pesar del valor económico de la especie, existen escasos antecedentes científicos relacionados con su reproducción controlada y cría en cautiverio para fines de cultivo comercial y/o repoblamiento. Algunos estudios en esta línea han sido realizados en Nueva Zelanda (Mitchell 1989) y en Chile (Barile et al. 2003).

Para la producción controlada de ovas y larvas de peces, es imprescindible conocer las condiciones ambientales físicas y químicas que influyen en la supervivencia de los organismos en cautiverio. En el caso de los peces que desarrollan parte de su ciclo de vida en ambientes estuarinos, como es el caso de la población migratoria de G. maculatus, la salinidad y la temperatura son las variables ambientales más influyentes en el metabolismo y desarrollo embrionario (Mihelakakis \& Yoshimatsu 1998, Ottesen \& Bolla 1998, Bhattacharya et al. 2006, Hassell et al. 2008, Peterei et al. 2008, Abdo-de la Parra et al. 2012).

La población migratoria de esta especie posee gran capacidad osmorregulatoria (Chesman \& Williams 1975) y migra desde el agua dulce hacia los estuarios donde desova entre la vegetación ribereña en periodos de altas mareas quedando los embriones expuestos a variaciones de salinidad y temperatura (Campos 1973, McDowall \& Robertson 1975, McDowall 1988, Berra et al. 1996,
McDowall 2001, McDowall \& Charteris 2006). Debido a lo anterior se hace necesario conocer el efecto de la salinidad y la temperatura así como el potencial efecto combinado que pueden ejercer estas 2 variables sobre el desarrollo embrionario de G. maculatus. La hipótesis del presente trabajo fue que al desarrollarse el embrión en el estuario sería capaz de tolerar salinidades desde 0 a 30 y temperaturas desde 5 a $15^{\circ} \mathrm{C}$. Junto con el objetivo de determinar el efecto de la temperatura y la salinidad en la supervivencia embrionaria de G. maculatus y su efecto en la viabilidad larval durante los primeros días post eclosión.

\section{Materiales Y MÉTODOS}

\section{Muestras biológicas}

Se utilizaron ovas de reproductores silvestres de Galaxias maculatus capturados en el estuario de Hornopirén, Chile ( $\left.41^{\circ} 52^{\prime} 34^{\prime \prime S}, 72^{\circ} 25^{\prime} 53^{\prime \prime O}\right)$ acondicionados en estanques en tierra de $600 \mathrm{~m}^{3}$ con tasa de recambio de agua dulce de $5 \%$ diario proveniente de una vertiente. Los peces se mantuvieron a una densidad de 0,3 a $0,4 \mathrm{~kg} \mathrm{~m}^{-3}$, alimentados con dietas extruidas para salmones (EWOS micro) una vez al día y a una ración aproximada de 1,5\% de su biomasa. Un total de 10 hembras maduras fueron desovadas manualmente mediante masaje abdominal, según la técnica descrita por Graaf \& Janssen (1996). Posteriormente las ovas fueron fertilizadas artificialmente 
con un pool de semen de 20 machos maduros mediante el método seco. La madurez sexual de los especímenes se determinó mediante una escala macroscópica de madurez sexual (Barile et al. 2003).

Previo al tratamiento de incubación los embriones fueron mantenidos por 3 días en placas Petri con agua de 10 ups, preparada a partir de agua dulce y sal de mar comercial, con recambio total del agua de una vez día, fotoperiodo de luz natural y temperatura constante de 10 $\pm 0,5^{\circ} \mathrm{C}$ fijadas mediante el uso de equipos de baño termorregulador, hasta el comienzo del experimento.

\section{DiSEÑo EXPERIMENTAL}

Para el presente experimento se evaluaron 3 niveles de temperatura: 5,10 y $15 \pm 0,5^{\circ} \mathrm{C}$, y 3 niveles de salinidad: 0 (dulce), 10 (salobre) y 30 (marina) $\pm 0,2$ ups. El experimento, tanto para embriones y larvas, se realizó 3 veces en distintos tiempos con 3 réplicas cada vez por tratamiento, empleando distintos grupos de reproductores.

Al tercer día post fertilización, mediante microscopio estereoscópico, fueron seleccionados los embriones que presentaban el disco embrionario en desarrollo, luego fueron incubados en placas Petri en grupos de 100 ovas a las diferentes combinaciones de temperatura y salinidad, previa aclimatación de los embriones a una tasa de 2 ups $\mathrm{h}^{-1} \mathrm{y}$ de $2^{\circ} \mathrm{C} \mathrm{h}^{-1}$.

Para cuantificar el efecto de la temperatura y salinidad en los embriones se determinó la supervivencia embrionaria que correspondió a la diferencia entre el número total de embriones al inicio del periodo experimental y el número total de embriones eclosionados al final de dicho periodo, expresados en porcentaje día.

Para evaluar el efecto de los tratamientos en la supervivencia de las larvas se mantuvieron grupos de 50 larvas recién eclosionadas por cada tratamiento en vasijas de vidrio de $1 \mathrm{~L}$ a una densidad de 10 larvas $\mathrm{L}^{-1}$, con agua de pozo a temperatura promedio de $10,7 \pm 1,1^{\circ} \mathrm{C}, 10 \mathrm{de}$ salinidad, aireación constante, recambio del $50 \%$ del volumen de agua una vez al día y alimentadas ad libitum con rotíferos (Brachionus plicatilis) enriquecidos a una concentración de 10 rotíferos $\mathrm{ml} \mathrm{día}^{-1}$. Para el caso de las larvas provenientes de los tratamientos con 10 ups y $10^{\circ} \mathrm{C}$, se aclimataron a una tasa de 2 ups $\mathrm{h}^{-1}$ y de $2^{\circ} \mathrm{C} \mathrm{h}^{-1}$. Cada tratamiento se realizó con 3 réplicas determinando la supervivencia larval a los 10 días post eclosión que correspondió a la diferencia entre el número total de larvas al inicio del experimento y el número total de larvas sobrevivientes al décimo día expresado en porcentaje.

\section{Análisis estadísticos}

Para determinar el efecto de la temperatura, la salinidad y su interacción en el desarrollo embrionario se empleó un ANDEVA factorial de $2 \times 2$ con 3 réplicas por experimento. Para el caso de la sobrevivencia larval se empleo un ANDEVA de una vía (Sokal \& Rohlf 1980). Las diferencias estadísticas entre las medias de los tratamientos fueron testadas al nivel de 0,05 utilizando la prueba de Duncan. Para satisfacer las suposiciones de normalidad del ANDEVA, los datos porcentuales fueron transformados mediante arco seno (Steel \& Torrie 1988). La normalidad de los datos se determinó mediante la prueba de Shapiro-Wilks y la homocedasticidad de su varianza mediante la prueba de Levene. Los análisis anteriormente señalados se realizaron empleando el software SPSS 20.

\section{Resultados Y Discusión}

A temperatura constante de $5^{\circ} \mathrm{C}$ se interrumpió la división celular del embrión dentro de las primeras 24 h y provocó $100 \%$ de mortalidad en todas las combinaciones de salinidades empleadas. Del mismo modo los embriones sometidos a salinidad constante de 30 , indistintamente de la temperatura empleada, evidenciaron desarrollo embrionario en los 5 primeros días; luego todos los embriones murieron (100\% de mortalidad). En las otras combinaciones de temperaturas de 10 y $15^{\circ} \mathrm{C}$ y salinidades de 0 y 10 los embriones completaron su desarrollo embrionario.

Las supervivencias embrionarias en agua dulce fueron de 76,0 y $75,1 \%$ a 10 y $15^{\circ} \mathrm{C}$ respectivamente, valores menores en comparación a los embriones en agua salobre, en donde se obtuvieron supervivencias de 87,1 y $85,4 \%$ a 10 y $15^{\circ} \mathrm{C}$ respectivamente, siendo significativamente diferentes en ambos grupos $\left(\mathrm{F}_{(1,35)}=56,890, P<0,05\right)$. Para el caso de la temperatura la supervivencia embrionaria no varió significativamente a 10 y $15^{\circ} \mathrm{C}\left(\mathrm{F}_{(1,35)}=0,808, P>\right.$ $0,05)$. No hubo efecto de la interacción temperaturasalinidad en la supervivencia de los embriones, $\left(\mathrm{F}_{(1,35)}=\right.$ $0,075, P>0,05)$ puesto que la salinidad afectó por igual a los embriones indistintamente de la temperatura empleada (Tabla 1).

Las mayores supervivencias larvales observadas a los 10 días de eclosionadas fueron de 88,7 y $80,0 \%$ a temperaturas de 10 y $15^{\circ} \mathrm{C}$ respectivamente y a salinidad de 10. Ambos valores de supervivencia fueron significativamente distintos entre sí. En cambio las larvas provenientes de embriones incubados en agua dulce 
Tabla 1. Supervivencia embrionaria promedio (expresados en porcentaje) y desviación estándar (entre paréntesis) de Galaxias maculatus para cada tratamiento de temperatura-salinidad / Mean embryonic survival (expressed as percentages) and standard deviation (between parentheses) of Galaxias maculatus per each temperature-salinity treatment

\begin{tabular}{cllll}
\hline Experimento & $10^{\circ} \mathrm{C}-0$ ups & $10^{\circ} \mathrm{C}-10$ ups & $15^{\circ} \mathrm{C}-0$ ups & $15^{\circ} \mathrm{C}-10$ ups \\
\hline 1 & $76,7(1,5)$ & $87,0(6,1)$ & $74,0(8,7)$ & $85,0(4,4)$ \\
2 & $75,7(2,1)$ & $87,3(5,0)$ & $77,3(2,1)$ & $86,7(5,9)$ \\
3 & $75,7(6,7)$ & $87,0(3,6)$ & $74,0(3,6)$ & $84,7(1,5)$ \\
Promedio & $76,0(3,61)^{(\mathrm{a})}$ & $87,1(4,34)^{(\mathrm{b})}$ & $75,1(5,11)$ & $85,4(3,84)$ \\
\hline
\end{tabular}

alcanzaron menores promedios de supervivencia, 74,7 y $69,3 \%$ a temperaturas de 10 y $15^{\circ} \mathrm{C}$ respectivamente, sin diferencias significativas entre ellas $\left(\mathrm{F}_{(3,32)}=9,05, P>\right.$ 0,05) (Tabla 2). Estos resultados concordaron a lo observado con la supervivencia embrionaria donde a salinidades de 10 se obtuvieron las más altas supervivencias.

El adulto de Galaxias maculatus es diádromo con gran capacidad osmorreguladora (Chesman \& Williams 1975) para vivir en ambientes de agua dulce y marina, sin embargo en el presente estudio sus embriones murieron al exponerlos a 30 ups constante, en cambio sobreviven en agua dulce y salobre, antecedentes que evidencian que su desplazamiento a los estuarios para desovar es parte de su estrategia reproductiva. A diferencia de lo anterior Young \& Dueñas (1993) señalan que los embriones de Siganus guttatus, que también es un pez diádromo, toleran amplios rangos de salinidad (entre 10 y $60)$, sin que este factor influya en los porcentajes de eclosión. Mientras que en la especie anádroma Takifugu obscurus, sus embriones toleran un margen estrecho de salinidad inferior a 12 (Yang \& Chen 2006).

La supervivencia promedio de los embriones de puye incubados en agua dulce y salobre fueron mayores al $75 \%$. Sin embargo, la supervivencia embrionaria en agua dulce es significativamente menor en comparación a las ovas incubadas a 10 ups. Probablemente las mayores supervivencias embrionarias en aguas salobres se relacionan con la conducta de la especie por migrar a zonas estuarinas en donde encuentra las salinidades óptimas para realizar exitosamente el desove y supervivencia de los embriones.

Varios estudios han demostrado que la interacción de la temperatura-salinidad influencian tanto el desarrollo embrionario como larval (Berlinsky et al. 2004, GraciaLopez et al. 2004, Cook et al. 2005, Nissling, et al. 2006, Brown et al. 2011, Ostrowski et al. 2011), sin embargo, en
Tabla 2. Supervivencia larval promedio (expresados en porcentaje) y desviación estándar (entre paréntesis) a los 10 días post-eclosión de Galaxias maculatus para cada tratamiento de temperaturasalinidad / Larval survival (expressed as percentages) and standard deviation (between parentheses) to 10 days post eclosion of Galaxias maculatus per each temperature-salinity treatment

\begin{tabular}{cllll}
\hline Experimento & $10^{\circ} \mathrm{C}-0$ ups & $10^{\circ} \mathrm{C}-10$ ups & $15^{\circ} \mathrm{C}-0$ ups & $15^{\circ} \mathrm{C}-10$ ups \\
\hline 1 & $76,0(8,2)$ & $87,7(12,9)$ & $68,3(7,6)$ & $78,7(8,7)$ \\
2 & $75,0(8,5)$ & $87,3(11,0)$ & $71,0(7,8)$ & $83,0(10,4)$ \\
3 & $73,0(9,0)$ & $91,0(7,6)$ & $68,7(8,4)$ & $78,3(9,9)$ \\
Promedio & $74,7(7,6)^{\text {(ac) }}$ & $88,7(9,5)^{(\mathrm{b})}$ & $69,3(7,0)^{(\mathrm{a})}$ & $80,0(8,7)^{(\mathrm{c})}$ \\
\hline
\end{tabular}

el presente estudio no se evidenció esta interacción en el desarrollo embrionario y posiblemente se requiera evaluar nuevos rangos de temperatura-salinidad.

En relación a la temperatura, los resultados indicaron que la supervivencia embrionaria de G. maculatus fue igualmente exitosa tanto en temperatura de $10^{\circ} \mathrm{C}$ como de $15^{\circ} \mathrm{C}$, sin diferencias significativas entre ellas. Se esperaba que los embriones tuviesen un rango de tolerancia restringido sólo a temperaturas frías, considerando su distribución sub antártica; sin embargo, la exposición de los embriones a temperatura constante de $5^{\circ} \mathrm{C}$, en los 3 niveles de salinidad provocó $100 \%$ de mortalidad. A diferencia de lo anterior hay especies de aguas frías que toleran temperaturas muy bajas, por ejemplo, la supervivencia embrionaria de Salvelinus alpinus es mayor a temperaturas de $3^{\circ} \mathrm{C}$ que a $6^{\circ} \mathrm{C}$ (De March 1995), otros resultados similares han sido observados en varias especies de la familia Salmonidae (Dwyer 1987, Tang et al. 1987, Wagner et al. 2006, Geist et al. 2008, Syrjanen et al. 2008, Teletchea et al. 2009).

En relación a la salinidad, Ottesen \& Bolla (1988) y Smith et al. (1999) demostraron que los embriones de Hippoglossus hippoglossus y Paralichthys lethogstima, son capaces de mantener su osmolaridad en diferentes ambientes salinos debido a que son impermeables a los solutos externo, pero al momento de eclosionar las larvas no sobreviven a salinidades bajas. Lo anterior también es planteado en los trabajos de Kucera et al. (2002) y Abdo de la Parra et al. (2012), quienes sostienen que varios de los órganos internos de las larvas al momento de eclosionar no son funcionales y ven disminuidas sus capacidades osmorregulatorias. En el presente estudio no hubo mortalidad al momento de la eclosión en las 2 salinidades empleadas demostrándose que la larva posee capacidad osmorregulatoria para soportar salinidades entre 0 y 10 , con supervivencia larval a los 10 días post eclosión superior al $69 \%$. 
En conclusión el desarrollo embrionario de $G$. maculatus es exitoso en agua dulce y salobre, en cambio el embrión es incapaz de desarrollarse en agua marina. Las combinaciones de 10 y $15^{\circ} \mathrm{C}$ a salinidad de 10 fueron donde se obtuvieron las más altas supervivencias embrionarias promedios $(>85 \%)$.

\section{Agradecimientos}

Los autores expresan sus agradecimientos a la empresa Piscicultura Pulelfu S.A. por financiar este proyecto y avanzar hacia la diversificación de la acuicultura nacional.

\section{LITERATURA CITADA}

Abdo-de la Parra I, I Martinez-Rodriguez, B GonzálezRodriguez, E Rodriguez-Ibarra, N Duncan \& C Hernández. 2012. Efecto de la temperatura y salinidad del agua en la incubación de huevos de botete diana Sphoeroides annulatus. Revista de Biología Marina y Oceanografía 47(1): 147-153.

Barile J, A Borquez, P Dantagnan, A Mardones, J Quevedo, I Salgado, I Valdebenito \& R Vega. 2003. Antecedentes para el cultivo del puye Galaxias maculatus (Pisces:Galaxiidae), 144 pp. Universidad Católica de Temuco, Temuco.

Benzie V. 1968. Stages in the normal development of Galaxias maculatus attenuatus (Jenyns). New Zealand Journal of Marine Freshwater Research 2: 606-627.

Berlinsky D, J Taylor, R Howell, T Bradley \& T Smith. 2004. The effects of temperature and salinity on early life stages of black sea bass Centropristis striata. Journal of the World Aquaculture Society 35(3): 335-344.

Berra T, L Crowley, W Ivantsoff \& P Fuerst. 1996. Galaxias maculatus: An explanation of its biogeography. Marine and Freshwater Research 47(6): 845-849.

Bhattacharya H, S Zhang, Y Wang \& Y Xu. 2006. Effects of salinity on embryogenesis and hatching of the rosy barb Puntius conchonius Hamilton 1822 (Cyprinidae). Tropical Zoology 19(1): 111-118.

Brown C, C Gothreaux \& C Green. 2011. Effects of temperature and salinity during incubation on hatching and yolk utilization of gulf killifish Fundulus grandis embryos. Aquaculture 315(3-4): 335-339.

Campos H. 1973. Migration of Galaxias maculatus (Jenyns) (Galaxiidae, Pisces) in valdivia estuary, chile. Hydrobiologia 43(3-4): 301-312.

Chessman B \& W Williams. 1975. Salinity tolerance and osmoregulatory ability of Galaxias maculatus (Jenyns) (Pisces, Salmoniformes, Galaxiidae). Freshwater Biology 5: $135-140$.
Cook M, K Guthrie, M Rust \& P Plesha. 2005. Effects of salinity and temperature during incubation on hatching and development of lingcod Ophiodon elongatus girard, embryos. Aquaculture Research 36(13): 1298-1303.

De March B. 1995. Effects of incubation temperature on the hatching success of arctic char eggs. The Progressive Fish Culturist 57: 132-136.

Dwyer W. 1987. Effect of lowering water temperature on hatching time and survival of lake trout eggs. The Progressive Fish-Culturist 49: 175-176.

Geist D, E Arntzen, C Murray, K McGrath, Y Bott \& T Hanrahan. 2008. Influence of river level on temperature and hydraulic gradients in chum and fall chinook salmon spawning areas downstream of bonneville dam, columbia river. North American Journal of Fisheries Management 28(1): 30-41.

Graaf G \& H Janssen. 1996. Artificial reproduction and pond rearing of the African catfish Clarias gariepinus in subSaharian-Africa - A handbook. FAO Fisheries Technical Paper 362: 1-73.

Gracia-Lopez V, M Kiewek \& M Maldonado. 2004. Effects of temperature and salinity on artificially reproduced eggs and larvae of the leopard grouper Mycteroperca rosacea. Aquaculture 237(1-4): 485-498.

Hassell K, P Coutin \& D Nugegoda. 2008. Hypoxia, low salinity and lowered temperature reduce embryo survival and hatch rates in black bream Acanthopagrus butcheri (Munro, 1949). Journal of Fish Biology 72(7): 1623-1636.

McDowall R. 1988. Diadromy in fishes. Migrations between freshwater and marine environments, 308 pp. Timber Press, Portland.

McDowall R. 2001. Diadromy, diversity and divergence: Implications for speciation processes in fishes. Fish and Fisheries 2: 278-285.

McDowall R \& D Robertson. 1975. Occurrence of galaxiid larvae and juveniles in the sea New Zealand Journal of Marine and Freshwater Research 9(1): 1-9.

McDowall R \& S Charteris. 2006. The possible adaptive advantages of terrestrial egg deposition in some fluvial diadromous galaxiid fishes (Teleostei:Galaxiidae). Fish and Fisheries 7(3): 153-164.

Mihelakakis A \& T Yoshimatsu. 1998. Effects of salinity and temperature on incubation period, hatching rate and morphogenesis of the red sea bream. Aquaculture International 6: 171-177.

Mitchell C. 1989. Laboratory culture of Galaxias maculatus and potential applications. New Zealand Journal of Marine \& Freshwater Research 23(3): 325-336.

Nissling A, U Johansson \& M Jacobsson. 2006. Effects of salinity and temperature conditions on the reproductive success of turbot (Scophthalmus maximus) in the Baltic Sea. Fisheries Research 80(2-3): 230-238. 
Ostrowski A, W Watanabe, F Montgomery, T Rezek, T Shafer \& J Morris. 2011. Effects of salinity and temperature on the growth, survival, whole body osmolality, and expression of na+/k+ atpase mrna in red porgy (Pagrus pagrus) larvae. Aquaculture 314(1-4): 193201.

Ottesen O \& S Bolla. 1998. Combined effects of temperature and salinity on development and survival of atlantic halibut larvae. Aquaculture International 6(2): 103-120.

Peterei C, H Haslob, G Kraus \& C Clemmesen. 2008. The influence of temperature on the development of Baltic Sea sprat (Sprattus sprattus) eggs and yolk sac larvae. Marine Biology 154: 209-306.

Sokal R \& J Rohlf. 1980. Introducción a la bioestadística, 362 pp. Reverté, Barcelona.

Steel R \& J Torrie. 1988. Bioestadística: Principios y procedimientos, 622 p. McGraw-Hill, New York.

Syrjanen J, M Kiljunen, J Karjalainen, A Eloranta \& T Muotka. 2008. Survival and growth of brown trout Salmo trutta. Embryos and the timing of hatching and emergence in two boreal lake outlet streams. Journal of Fish Biology 72(4): 985-1000.
Tang J, M Bryant \& E Brannon. 1987. Effect of temperature extremes on the mortality and development rates of coho salmon embryos and alevins. The Progressive Fish Culturist 49: $167-174$

Teletchea F, J Gardeur, E Kamler \& P Fontaine. 2009. The relationship of oocyte diameter and incubation temperature to incubation time in temperate freshwater fish species. Journal of Fish Biology 74(3): 652-668.

Wagner E, R Arndt \& R Roubidoux. 2006. The effect of temperature changes and transport on cutthroat trout eggs soon after fertilization. North American Journal of Aquaculture 68(3): 235-239.

Yang Z \& Y Chen. 2006. Salinity tolerance of embryos of obscure puffer Takifugu obscurus. Aquaculture 253(1-4): 393-397.

Young P \& C Dueñas. 1993. Salinity tolerance of fertilized eggs and yolk-sac larvae of the rabbitfish Siganus guttatus (bloch). Aquaculture 112(4): 363-377.

Recibido el 6 de noviembre de 2012 y aceptado el 30 de agosto de 2013

Editor Asociado: Gabriela Muñoz C. 